\title{
Linear viscoelastic properties of hyperbranched polyisobutylene
}

\author{
C. G. Robertson and C. M. Roland ${ }^{\mathrm{a})}$ \\ Chemistry Divison, Code 6120, Naval Research Laboratory, \\ Washington, D.C. 20375-5342 \\ C. Paulo and J. E. Puskas \\ Department of Chemical \& Biochemical Engineering, University of Western \\ Ontario, London, Ontario N6A 5B9, Canada
}

(Received 27 September 2000; final revision received 18 January 2001)

\section{Synopsis}

Creep and dynamic mechanical measurements were carried out on a series of hyperbranched polyisobutylenes (PIBs), having a range of molecular weights ( $\geqslant 10^{6}$ daltons) and branching frequencies (3-57 branches/molecule). For all samples, the molecular weight of the branches was higher than the entanglement molecular weight of linear PIB, by as much as a factor of 10; nevertheless, only for molecular weights of approximately half-million daltons does the zero-shear viscosity exceed that of linear PIB. Both the viscosity and the length of the entanglement plateau are governed primarily by the branching frequency, rather than by the length of the branches. Such behavior is quite distinct from star-branched polymers. However, the magnitude of the plateau modulus and the temperature dependence of the terminal zone shift factors are the same for hyperbranched, star-branched, and linear PIB. (C) 2001 The Society of Rheology.

[DOI: $10.1122 / 1.1357821]$

\section{INTRODUCTION}

The molecular architecture of polymers governs to a significant extent their physical properties, leading to widespread interest in the study of branched polymers. The rheology is especially sensitive to the presence of long branches, as shown in the pioneering work of Long et al. (1964) and Kraus and Gruver (1965), and reviewed by various authors [Grest et al. (1996); Bauer and Fetters (1978); Graessley (1977)]. There are two principal factors [Berry and Fox (1968)]. The first is the effect branching has on the molecular size, which can be as expressed as the ratio of the mean square radius of gyration $\left\langle R_{G}^{2}\right\rangle$ of the branched chain to that for the linear polymer having the same molecular weight

$$
g=\frac{\left\langle R_{G}^{2}\right\rangle}{\left\langle R_{G}^{2}\right\rangle_{\text {lin }}},
$$

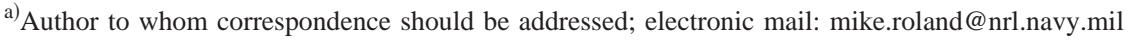


with $\left\langle R_{G}^{2}\right\rangle$ often determined by light scattering. Branching reduces the size $(g<1)$, which enhances molecular mobility, aside from any effect on topological interactions.

The second effect, first pointed out by Long et al. (1964), is that branches hinder long-range, cooperative motions of the chains, making entanglements more effective. This property has long been taken advantage of to suppress cold flow (room temperature creep during storage) of rubber [White (1969)]. Long chain branching also reduces the diffusion constant and gives rise to a dependence of the zero-shear viscosity $\eta_{0}$ on molecular weight that exceeds the 3.4 power law found for linear polymers [Fetters et al. (1993); Grest et al. (1996); Ngai and Roland (1997)]. For star polymers, in which all branches emanate from the same site, diffusion coefficients and viscosity depend more strongly on the molecular weight of the branch $M_{\mathrm{Br}}$ than on the number of branches $f$ [Shull et al. (1988); Fetters et al. (1993); Grest et al. (1996)].

Only a few, randomly situated long branches are necessary for substantial changes in the flow properties of polymers; hence, there is substantial interest in tailoring chain topologies to optimize processability. For comparisons to theory and the development of structure-property relationships, star polymers are attractive. The size of a star molecule having equal length arms is a unique function of $f$ [Zimm and Stockmayer (1949); Graessley (1977)], and predictions are available concerning the properties of uniform star polymers [Pearson (1987); Grest et al. (1996)]. More complicated branched structures, such as combs [Roovers and Graessley (1981); Roovers and Toporowski (1987)], miktoarm stars [Hadjichristidis (1999); Hutchings and Richards (1998)], H polymers [Roovers (1984); McLeish et al. (1999)] and the related pom-pom polymers [McLeish and Larson (1998); Inkson et al. (1999)], can also serve as model polymers.

Polymers with greater deviations from a linear topology, rings [McKenna et al. (1989); Tead et al. (1992)] and microgel polymers [Antonietti et al. (1995); Roland et al. (1996, Roland et al. 1999); McGrath et al. (2000)], are of interest because they lack intermolecular entanglements, yet their low frequency dynamics are quite similar to that of linear polymer melts. Another type of material with reduced intermolecular constraints is dendrimers. Inspired by biomolecules, dendrimers are comprised of treelike branches issuing from a common core [Hawker (1999); Frechet (1994)]. The crowding at the molecular periphery precludes entanglements, leading to Rouse-like behavior, even at molecular weights as high as $10^{5}$ [Farrington et al. (1998); Hawker et al. (1995)]. Roovers and co-workers [Roovers et al. (1993); Zhou et al. (1992)] used a dendrimer coupling agent to synthesize highly branched chains $(f>24)$, with somewhat opposite behavior. The core region of the molecule is densely packed and impenetrable by neighboring chains materials, which causes an order to develop in the melt [Pakula and coworkers (1998)] and leads to heterogeneity of the molecular motions [Kwak and Lee (2000)]. Related to these structures are "poly(macromonomers)," which have a high density of branches attached to a central chain backbone [Namba et al. (2000)].

The present paper is concerned with a material having another type of branch structure, hyperbranched polyisobutylene (PIB). The chains have profuse branches in a treelike structure, with the branches extending between successive tiers (Fig. 1). However, the surface regularity of dendrimers is lacking. Unlike star polymers, not all branches in hyperbranched PIB are dangling (unattached at one end). Star-branched PIB has been the focus of recent research [Santangelo et al. (1999); Jacob and Kennedy (1999); Puskas and Wilds (1998); Puskas et al. (1999); Storey et al. (1997)]. Herein we describe rheological measurements, intended to examine how linear viscoelastic properties may be influenced by the three-dimensional structure. 


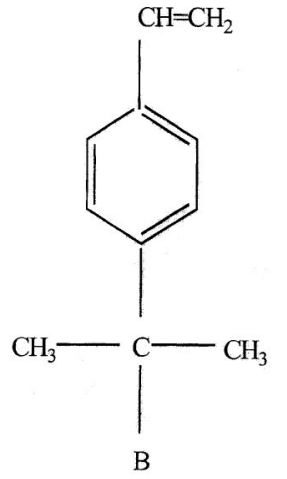

$\mathrm{B} \equiv$ isobutylene

(a)

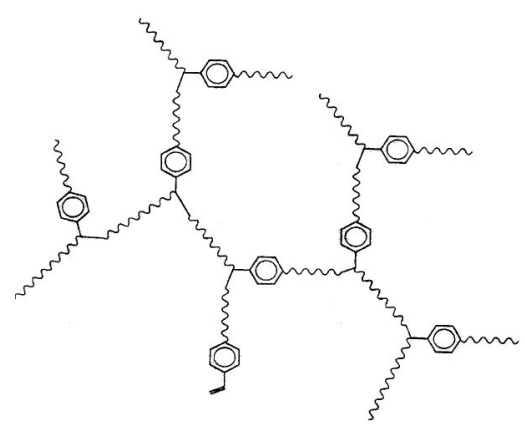

(b)

FIG. 1. Structure of (a) inimer, which is copolymerized with isobutylene to yield (b) hyperbranched PIB.

\section{EXPERIMENT}

The hyperbranched PIB was synthesized by copolymerizing isobutylene and an "inimer," which combines monomer and initiator functions [Fig. 1(a)] [Puskas and Grassmuller (1998); Puskas and Paulo (2001)]. The samples encompass a range of branch structures and molecular weights, and have a branch functionality of 3 , independent of the branch frequency [Fig. 1(b)]. Molecular weights were measured by gel permeation chromatography (GPC) (Waters Corp.) coupled with multiangle laser light scattering (Wyatt Technology Corp.). The branch molecular weights were determined by GPC after selective cleavage at the aromatic ring linkages, using the method described by Kennedy et al. (1981). It is a significant advantage to use chemical means to characterize the branching. A well-known problem in studying the rheology of branched polymers is the difficulty in characterizing branching using traditional methods, such as spectroscopy [see, for example, Janzen and Colby (1999)]. The experimentally obtained branching frequencies were in good agreement with the values calculated from the inimer content. Samples are listed in Table I, identified by their weight average molecular weight and number average branch frequency ( $f=M_{n} / M_{\mathrm{Br}}$, in which $M_{\mathrm{Br}}$ is the number average); thus, for H1080_21, $M_{w}=1080 \times 10^{3}$ daltons and $f=21$ branches $/$ molecule.

Creep viscosities were measured with a Haake RS150 RheoStress instrument, using 8 $\mathrm{mm}$ diam parallel plates. Most tests were performed at $25^{\circ} \mathrm{C}$, with each measurement continued until terminal behavior was attained. In some cases, this required as much as 10 days. For the highest molecular weight samples, creep testing was carried out at $130^{\circ} \mathrm{C}$, with the viscosity for $25^{\circ} \mathrm{C}$ calculated using shift factors obtained from dynamic mechanical data. The reproducibility of the viscosity measurements was about $6 \%$.

Isothermal dynamic mechanical measurements were made over the frequency range from $5 \times 10^{-5}$ to $1 \mathrm{~Hz}$, at temperatures from -60 to $150^{\circ} \mathrm{C}$. For stiff samples and low temperatures, a Bohlin VOR equipped with a high torque transducer $(2000 \mathrm{~g} \mathrm{~cm}$ full scale) was used. Measurements on softer samples employed a second Bohlin instrument, which uses torsion bars having maximum torques in the range from 11 to $300 \mathrm{~g} \mathrm{~cm}$. The use of two instruments, in combination with several parallel plates having diameters from 6.4 to $40 \mathrm{~mm}$, allowed the large range of sample compliances to be accommodated. Figure 2 illustrates the reproducibility of data obtained from the different instruments, 
TABLE I. Macrostructure of polyisobutylenes.

\begin{tabular}{|c|c|c|c|c|c|c|}
\hline Designation & Structure & $\begin{array}{l}M_{w} \times 10^{-3} \mathrm{a} \\
\quad \text { (daltons) }\end{array}$ & $M_{w} / M_{n}{ }^{\mathrm{a}}$ & $\begin{array}{l}M_{\mathrm{Br}} \times 10^{-3 \mathrm{~b}} \\
\quad \text { (daltons) }\end{array}$ & $f^{\mathrm{c}}$ & $\begin{array}{c}\eta_{0}{ }^{\mathrm{d}} \\
(\mathrm{Pa} \mathrm{s})\end{array}$ \\
\hline L176 & linear & 176 & 1.6 & - & - & $8.9_{5} \times 10^{6}$ \\
\hline L389 & linear & 500 & 2.5 & - & - & $1.29 \times 10^{8}$ \\
\hline S217 & star & 217 & 1.2 & 36.2 & 6 & $6.8 \times 10^{6}$ \\
\hline S490 & star & 490 & 1.2 & 81.7 & 6 & $1.3_{2} \times 10^{8}$ \\
\hline H1080_21 & hyperbranched & 1,080 & 2.6 & 52.7 & 20.5 & $1.1_{1} \times 10^{8}$ \\
\hline H984_57 & hyperbranched & 984 & 1.3 & 17.2 & 57.2 & $2.8_{7} \times 10^{9}$ \\
\hline H790_44 & hyperbranched & 790 & 1.3 & 17.9 & 44.1 & $1.7_{7} \times 10^{9}$ \\
\hline H681_33 & hyperbranched & 681 & 1.2 & 20.6 & 33.0 & $6.27 \times 10^{8}$ \\
\hline H280_7 & hyperbranched & 280 & 3.0 & 37.7 & 7.4 & $5.0_{4} \times 10^{6}$ \\
\hline H275_8 & hyperbranched & 275 & 2.5 & 24.1 & 7.8 & $9.3_{3} \times 10^{5}$ \\
\hline $\mathrm{H} 265 \_12$ & hyperbranched & 265 & 1.9 & 23.1 & 11.5 & $7.9_{3} \times 10^{6}$ \\
\hline H236_9 & hyperbranched & 236 & 2.7 & 26.4 & 8.9 & $1.9_{2} \times 10^{6}$ \\
\hline H230_9 & hyperbranched & 230 & 2.1 & 23.5 & 9.2 & $2.3_{7} \times 10^{6}$ \\
\hline $\mathrm{H} 223 \_5$ & hyperbranched & 223 & 2.9 & 53.6 & 4.6 & $9.9_{2} \times 10^{5}$ \\
\hline $\mathrm{H} 217 \_4$ & hyperbranched & 217 & 2.2 & 42.4 & 4.1 & $3.4_{8} \times 10^{4}$ \\
\hline H180_8 & hyperbranched & 180 & 1.7 & 27.9 & 7.6 & $1.7_{5} \times 10^{6}$ \\
\hline H151_5 & hyperbranched & 151 & 2.1 & 27.8 & 5.4 & $2.5_{1} \times 10^{5}$ \\
\hline H144_7 & hyperbranched & 144 & 2.1 & 21.4 & 6.7 & $2.5_{2} \times 10^{5}$ \\
\hline H136_5 & hyperbranched & 136 & 2.3 & 26.4 & 5.2 & $2.1_{9} \times 10^{5}$ \\
\hline H130_7 & hyperbranched & 130 & 1.8 & 22.0 & 7.0 & $1.0_{5} \times 10^{6}$ \\
\hline H76_4 & hyperbranched & 76 & 1.8 & 18.8 & 3.5 & $7.3_{4} \times 10^{4}$ \\
\hline $\mathrm{H} 75 \_3$ & hyperbranched & 75 & 2.0 & 21.5 & 3.0 & $1.8_{4} \times 10^{4}$ \\
\hline
\end{tabular}

${ }^{\mathrm{a} M A L L S .}$

${ }^{\mathrm{b}} M_{w}$ of branch.

${ }^{\mathrm{c}}$ Number average.

$\mathrm{d}_{25}{ }^{\circ} \mathrm{C}$.

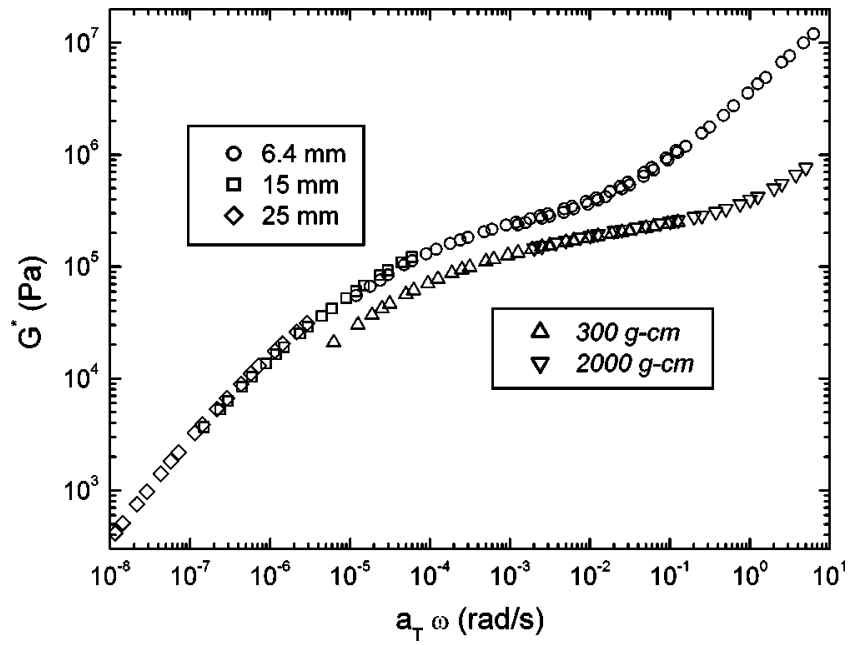

FIG. 2. Representative data from Bohlin rheometer affirming reproducibility of measurements: upper curve: on H230_9 obtained with parallel plates of the indicated diameter; lower curve: on H1080_21 obtained using different torque transducers. 


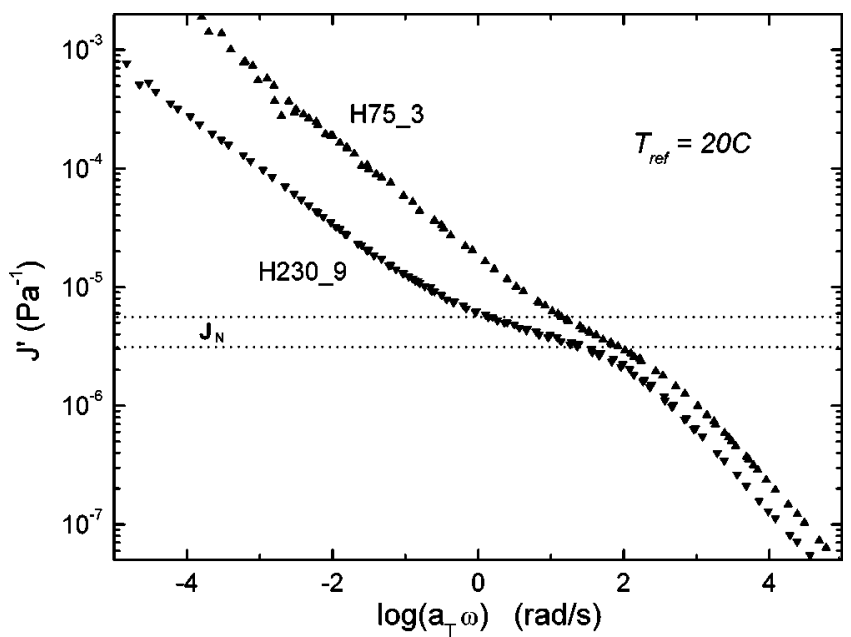

FIG. 3. Master curves of the storage compliance for two hyperbranched PIB, having equal branch lengths and equal molecular weight distributions (see Table I). However, H230_9 $(\boldsymbol{\nabla})$ has three times as many branches, and thus a threefold higher molecular weight, resulting in a lower compliance in comparison to H75_3 ( $\mathbf{\Delta})$. Note that although the polymers have entangled branches $\left(M_{\mathrm{Br}} / M_{e} \sim 2.4\right)$, there is no entanglement plateau. The horizontal lines indicate the highest [Plazek et al. (1992)] and lowest [Fetters et al. (1991)] literature values reported for the plateau compliance of linear PIB.

affirming the accuracy of the plateau moduli comparisons. Strain sweeps were conducted at each temperature to verify linearity. During a measurement, the strain was changed to maintain a constant torque as the frequency was swept. For both creep and dynamic mechanical measurements, sample gaps were in the range of $0.7-2 \mathrm{~mm}$.

\section{RESULTS AND DISCUSSION}

Master curves were constructed from isothermal dynamic mechanical measurements at various temperatures, with the frequency-temperature shift factors obtained by empirical superposition of the loss tangent. Figure 3 shows the storage compliance $J^{\prime}(\omega)$, where $\omega$ is angular frequency, for two hyperbranched PIB. H230_9 and H75_3 have equal branch lengths and the same molecular weight distribution, but differ in their respective branch frequencies. We display the compliance function because it unambiguously reveals the presence of an entanglement network [Plazek and Echeverría (2000)]. A factor of 3 increase in the number branches per molecule is seen to increase the separation between the transition and terminal zones. However, there is no plateau evident in the spectra of Fig. 3, even though $M_{\mathrm{Br}}$ exceeds the entanglement molecular weight $M_{e}$ by more than a factor of 2. For linear PIB, $M_{e} \sim 9400$ daltons [Santangelo et al. (1999)], which corresponds to a plateau compliance $J_{N}=3.5 \mathrm{MPa}^{-1}$; the range of literature values for PIB [Plazek et al. (1992); Fetters et al. (1991)] is indicated by the horizontal lines in the figure. $M_{\mathrm{Br}}$ of these two polymers also exceeds the critical molecular weight for viscosity enhancement, which for PIB is only 60\% larger than $M_{e}$ [Graessley and Edwards (1981)].

A greater degree of branching reduces the compliance in the flow region. This effect is evident in the loss modulus $G^{\prime \prime}(\omega)$, displayed in Fig. 4 for these two samples. Although the dynamic measurements do not extend to sufficiently low frequency for $G^{\prime \prime} / \omega$ to become constant, it can be seen that the threefold increase in the number of branches, at constant $M_{\mathrm{Br}}$, increases the viscosity by 2 orders of magnitude. For comparison, the 


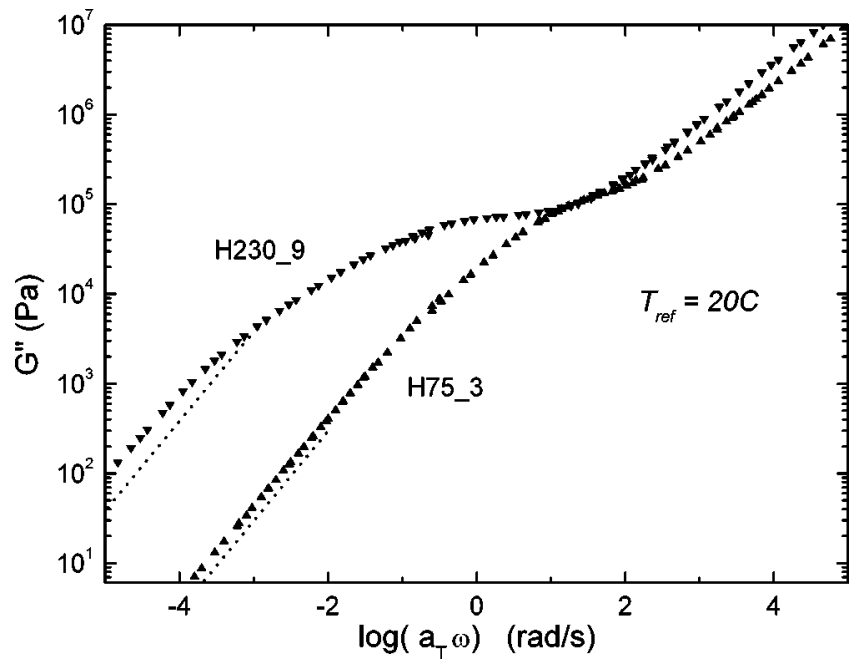

FIG. 4. Master curves for the loss modulus of the two hyperbranched PIB of Fig. 3, which have equal length branches $\left(M_{\mathrm{Br}} / M_{e} \sim 2.4\right)$. The dashed lines correspond to the product $\eta_{0} \times \omega$, where $\eta_{0}$ is the terminal viscosity determined by creep measurements.

zero-shear viscosities measured by creep for the two samples are included in the figure, after multiplication by $\omega$. The difference in viscosity between H230_9 and H75_3 substantially exceeds the 3.4 power dependence on molecular weight expected for linear polymers. In this strong dependence of their viscosity on the number of branches, the behavior of hyperbranched PIB is contrary to that generally found for star-branched polymers [Fetters et al. (1993); Grest et al. (1996)].

Zero-shear viscosities determined from creep experiments are displayed in Fig. 5 for all hyperbranched PIB in Table I which have a polydispersity less than 2.2. In the figure, we include the relation for linear PIB determined by Fetters et al. (1991)

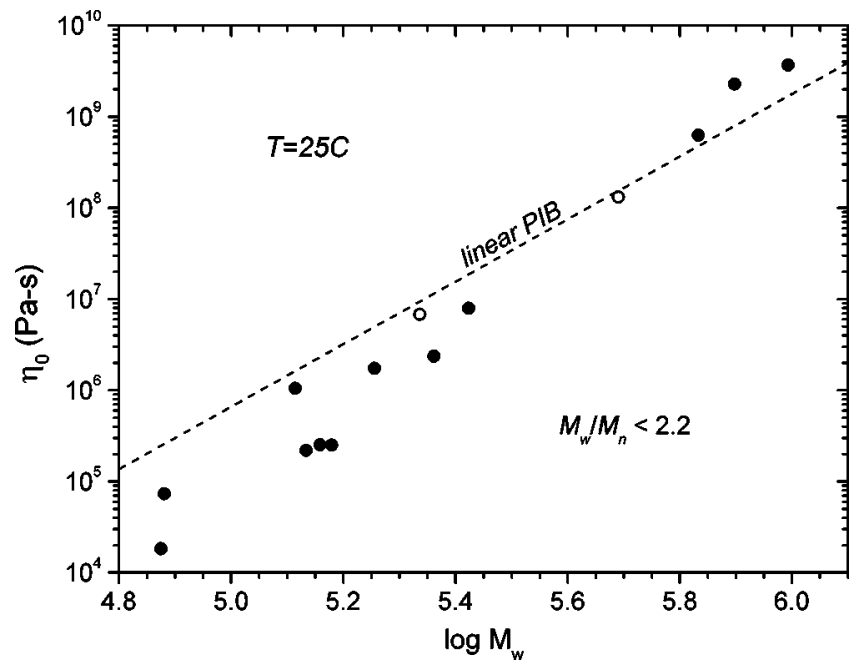

FIG. 5. Molecular weight dependence of the zero-shear viscosity for hyperbranched $(\bullet)$ and star-branched $(\bigcirc)$ PIB, along with Eq. (2) (- - ) for linear PIB. Only samples in Table I for which $M_{w} / M_{n}<2.2$ are included in this figure. 


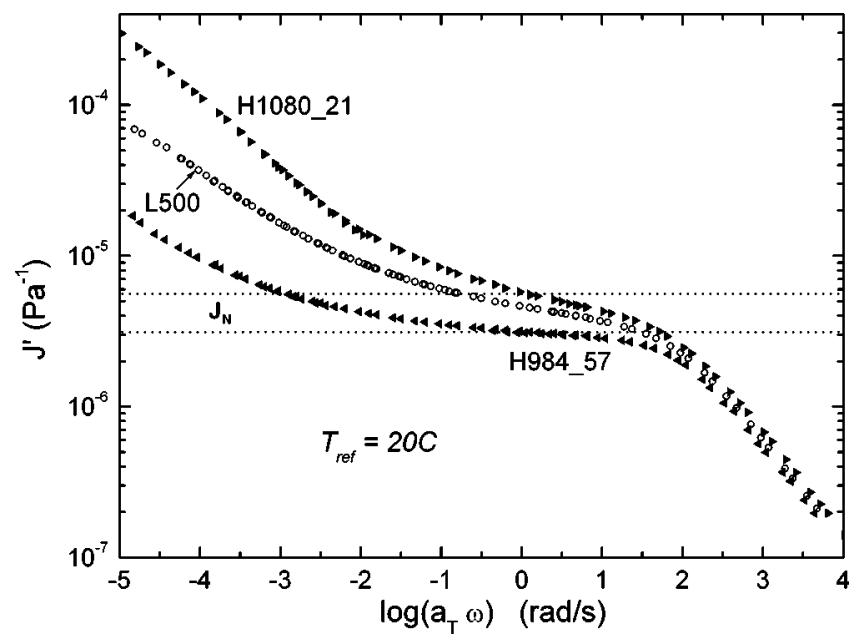

FIG. 6. Master curves of the storage compliance for two hyperbranched PIB of approximately equal molecular weight, and for a linear PIB. Although H1080_21 $(-)$ has longer branches $\left(M_{\mathrm{Br}} / M_{e} \sim 5.6\right)$, its entanglement plateau is less prominent than for H984_57 ( $)$, for which $\left(M_{\mathrm{Br}} / M_{e}<2\right)$. The plateau values for the hyperbranched polymers encompass the $J_{N}$ measured for L389 (O). The horizontal lines indicate the highest [Plazek et al. (1992)] and lowest [Fetters et al. (1991)] literature values for linear PIB.

$$
\eta_{0}=4.69 \times 10^{-12} M_{w}^{3.43}
$$

at $25^{\circ} \mathrm{C}$ in units of $\mathrm{Pas}$, with $M_{w}$ in daltons. The molecular weight range of the hyperbranched PIB is insufficient to determine the molecular weight dependence of the viscosity (i.e., exponential [Doi and Edwards (1986)] or something weaker [Ngai and Roland (1997); Janzen and Colby (1999)]). Only for molecular weights exceeding about a half million does $\eta_{0}$ exceed that of linear PIB. This is despite the fact that the samples in Fig. 4 have entangled branches, $1.8 \leqslant M_{\mathrm{Br}} / M_{e}<10$. Nevertheless, the dependence of their viscosity on molecular weight appears to be stronger than that given by Eq. (2). The molecular weight dependence for six-arm star PIB, reported by Santangelo et al. (1999) and included in Fig. 5, is indistinct from the behavior of the hyperbranched polymers.

Generally, it is considered that from two to several entanglements [Kraus and Gruver (1965); Graessley et al. (1976); Shull et al. (1991)] are necessary for viscosity enhancement. A greater degree of entanglement appears necessary for viscosity enhancement in the hyperbranched polymers, reflecting the different nature of their branches (i.e., mostly attached at both ends). When the branching density becomes very high, reduced intermolecular penetration can also exert an effect [Pakula and co-workers (1998); Kwak and Lee (2000)].

In Fig. 6 are storage compliance master curves for H1080_21 and H984_57, hyperbranched PIB having about the same $M_{w}$, with the former having longer branches while the latter has many more branches. H1080_21 also has a broader molecular weight distribution (Table I). In Fig. 6, H984_57 exhibits a prominent rubbery plateau, even though its branches are not highly entangled $\left(M_{\mathrm{Br}} / M_{e}=1.8\right)$. In fact, they are shorter than the branch lengths of the polymers in Fig. 3, for which a plateau in $J^{\prime}(\omega)$ is absent. Interestingly, the entanglement plateau of H1080_21 is less developed than that for H984_57, even though the former has a slightly higher molecular weight and more entangled branches $\left(M_{\mathrm{Br}} / M_{e}=5.6\right)$. For comparison, included in Fig. 6 is $J^{\prime}(\omega)$ measured for L389, a linear PIB. It's entanglement plateau is within the range of literature 


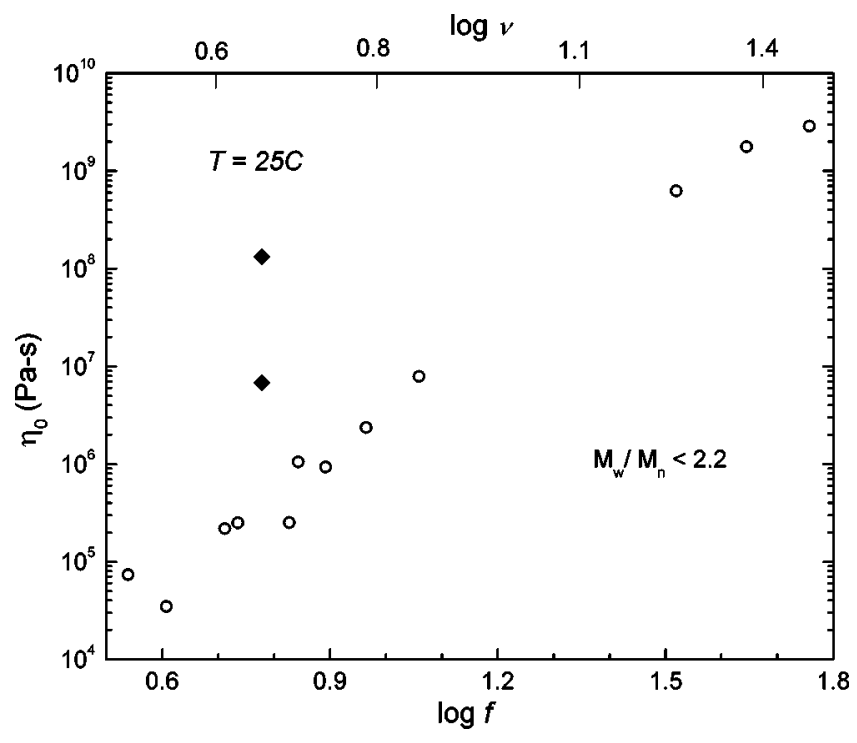

FIG. 7. Zero-shear rate viscosity of branched PIB as a function of the branching frequency. The relationship between these two quantities for hyperbranched PIB $(O)$ is quite different from that of star polymers, as seen in the data for six-arm PIB stars ( ) taken from Santangelo et al. (1999). The corresponding arm number [Eq. (3)] is shown on the upper axis. Only samples in Table I for which $M_{w} / M_{n}<2.2$ are included in this figure.

values for $J_{N}$ [Fetters et al. (1991); Plazek et al. (1992), Santangelo et al. (1999)], and intermediate to that of the hyperbranched polymers.

The zero-shear viscosity of H1080_21 is also smaller by a factor of 28 than $\eta_{0}$ for H984_57. It is striking that both $\eta_{0}$ and the magnitude of the entanglement plateau depend more strongly on the number of branches than on $M_{\mathrm{Br}}$. Moreover, this observation is not related to polydispersity in molecular weight, even though $M_{w} / M_{n}$ differ significantly for the two polymers. For example, comparing the three most monodisperse hyperbranched samples in Table I $\left(M_{w} / M_{n} \approx 1.3\right)$, their zero-shear viscosities rank order as H984_57 > H790_44 > H681_33. This follows exactly their branching frequencies, but is inversely related to their respective $M_{\mathrm{Br}}$.

This correlation between $\eta_{0}$ and branching frequency is the general pattern for the hyperbranched PIB (Fig. 7), notwithstanding variations of $M_{\mathrm{Br}}$. A zero-shear viscosity that is almost a unique function of $f$ is quite different behavior from star-branched polymers, as illustrated in Fig. 7 by the two data points for six-arm PIB stars. The obvious structural difference between hyperbranched and star polymers is that only for the latter is each branch a dangling chain (i.e., an arm). This difference can be quantified by approximating the hyperbranched structure as a Cayley tree [Bick and McLeish (1996); Atilgan et al. (1998)]. For trifunctional junctions (Fig. 1), the number of dangling chains $\nu$ is related to the branching frequency as [Janzen and Colby (1999)]

$$
\nu=\frac{f+3}{2} .
$$

The dependence of the viscosity on the number of arms (the latter given as the upper abscissa scale of Fig. 7) is quite similar to its dependence on the number of branches; thus, the quite different behavior of hyperbranched from star-branched PIB remains. 


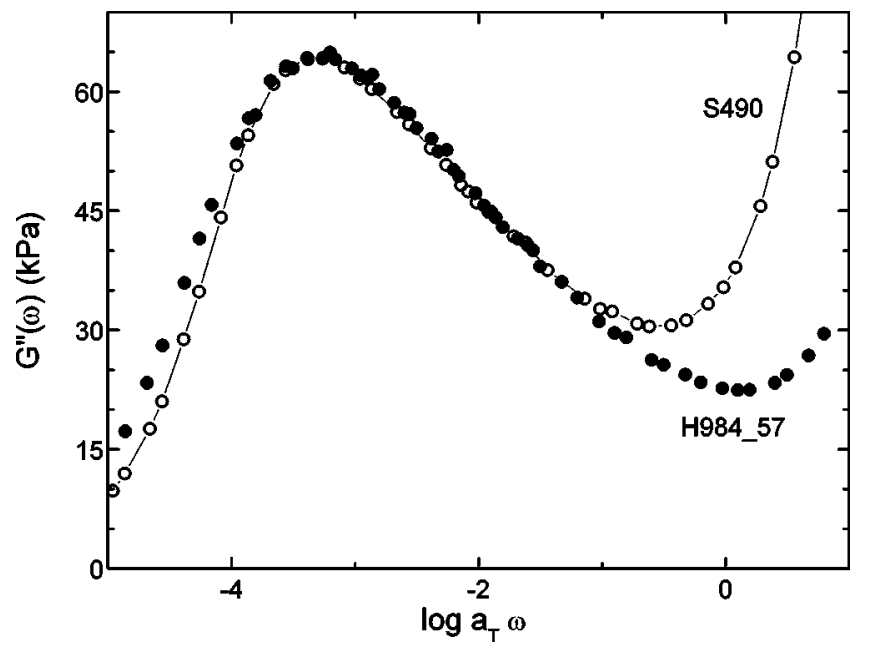

FIG. 8. Dispersion in the loss modulus for a hyperbranched PIB $(\bullet)$ at $T_{\text {ref }}=20^{\circ} \mathrm{C}$ and for a six-arm star PIB (○), taken from Santangelo et al. (1999). Frequencies for the latter have been divided by a factor of 134 to superpose the peaks.

The well-developed entanglement plateau in H984_57 gives rise to a dispersion in the loss modulus, displayed on a semilogarithmic scale in Fig. 8. Also included in the figure is the corresponding peak for S490, a six-arm star PIB having similar polydispersity $\left(M_{w} / M_{n} \approx 1.2\right)$. Their shapes are roughly comparable, although the shorter plateau in the viscoelastic spectrum of S490 causes greater overlapping with the transition zone. While theories for the shape of the modulus function (i.e., time dependence of the loss modulus) for branched molecules are available, they cannot quantitatively predict experimental results, especially toward higher frequencies. This is true not only for branched polymers [Pearson and Helfand (1984); Milner and McLeish (1997)], but even for linear chains [Doi and Edwards (1986)]. Constraint release is believed to be important for the terminal relaxation of branched polymers [Ball and McLeish (1989); Shull et al. (1991)], but theory therein is at odds with experiment, again even for linear polymers. This has been shown by experiments in which putative constraint release mechanisms are suppressed by blending [Adachi et al. (1995); Schroeder and Roland (1999).]. Thus, we eschew any analysis of the shape of the loss modulus peaks.

The effect of temperature on the rheological properties of branched polymers is a controversial topic. Early results on polyethylenes showed that long chain branching significantly increased the temperature coefficent of viscosity [Berry and Fox (1968); Graessley (1977)]. Graessley (1982) developed a model based on the idea that terminal relaxation of branched polymers involves retraction of oriented arms. Retraction of an arm requires a transient change in the trans/gauche population, leading to the prediction of a relationship between the trans/gauche energy difference and the effect of branching on the temperature coefficient. The model has meet with mixed success in accounting for experimental results on different star-branched polymers [Carella et al. (1986); Santangelo and Roland (1998); Bero and Roland (1996); Roland and Bero (1996)].

Polyisobutylene is an interesting case, because its trans and gauche conformers have virtually the same energy [Erman and Mark (1997), Suter et al. (1983)]. The consequent prediction from Graessley's (1982) arm retraction model, that the temperature coefficient for terminal relaxation will be equivalent for branched and linear PIB, is consistent with experiments of Santangelo et al. (1999) on six-arm star PIB. With the possible exception 


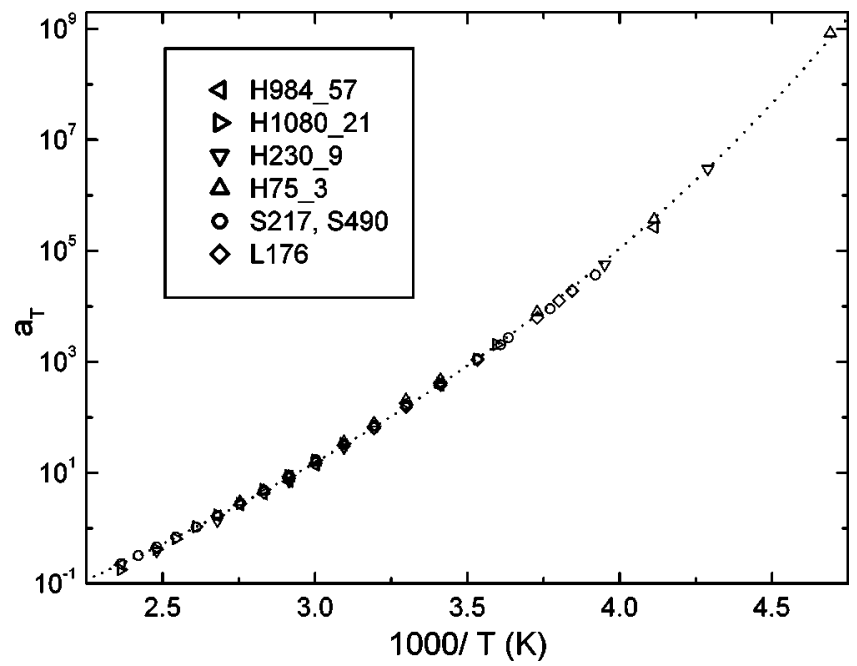

FIG. 9. The temperature dependence of the time-temperature shift factors or the zero-shear viscosities for hyperbranched, six-arm star, and linear polyisobutylenes. The dashed line through the data is the fit to Eq. (4) (see Table II).

of hydrogenated 1,2-polybutadiene [Carella et al. (1986)], PIB is the only case of a polymer with well-entangled branches having the same temperature dependence as its linear counterpart.

Time-temperature shift factors determined from dynamic mechanical measurements of the terminal zone for four hyperbranched PIB are shown in Fig. 9, along with data for two six-arm star PIB and a linear PIB [Santangelo et al. (1999)]. For the hyperbranched polymers, $1.8 \leqslant M_{\mathrm{Br}} / M_{e} \leqslant 5.6$, with as many as 57 branches per molecule. All species exhibit the same temperature dependence, which is noteworthy, since the effects of branching-dominated by $M_{\mathrm{Br}} / M_{e}$ for stars but more influenced by $f$ for hyperbranched PIB-are so diverse.

Fitting the data in Fig. 9 to the Vogel-Fulcher equation [Berry and Fox (1968)]

$$
a_{T} \propto \exp \left(\frac{b}{T-T_{\infty}}\right)
$$

yields $b=3170$ and $T_{\infty}=114 \mathrm{~K}$. We can compare this to literature values for linear PIB, reported using the mathematically equivalent Williams-Landel-Ferry (WLF) equation [Ferry (1980)]

$$
\log a_{T}=-\frac{C_{1}\left(T-T_{0}\right)}{C_{2}+T-T_{0}} .
$$

The respective parameters are related according to $b=2.303 C_{1} C_{2}$ and $T_{\infty}=T_{0}$ $-C_{2}$ [Ferry (1980)]. As seen in Table II, the present data are in good agreement with previous results for linear PIB [Ferry and Parks (1935); Berry and Fox (1968); Fetters et al. (1991)].

\section{CONCLUSIONS}

A principal finding herein is that the rheology of hyperbranched PIB is governed primarily by the number of branches per molecule, rather than by the length of the branches. High branching frequency is associated with both a large terminal viscosity and 
TABLE II. Temperature dependence of PIB in terminal zone.

\begin{tabular}{lccl}
\hline \hline \multicolumn{1}{c}{ PIB } & $b$ & $T_{\infty}(\mathrm{K})$ & \multicolumn{1}{c}{ Source } \\
\hline branched and linear & 3170 & 114 & This work \\
linear & 3220 & 114 & Ferry and Parks (1935) \\
linear & 3120 & 122 & Berry and Fox (1968) \\
linear & 3310 & 106 & Fetters et al. (1991) \\
\hline \hline
\end{tabular}

an extended entanglement plateau (spanning a longer time scale). However, at relatively low branch densities, the development of an entanglement plateau requires branch molecular weights more than a factor of 2 larger than $M_{e}$. This reflects in part the fact that internal branches (not dangling) can interact only via a loop, or "knitting," mechanism [MacArthur and Stephens (1983)], as opposed to the topological interactions of linear chains and star polymers, for which the ends are the active part [Doi and Edwards (1986)]. In this respect, hyperbranched PIB has similarities to polystyrene microgels, which are unentangled, high molecular weight polymers [Antonietti et al. (1995); Roland et al. (1996, 1999); McGrath et al. (2000)]. In contrast to the dynamic properties, the magnitude of the plateau compliance, a static property, for the hyperbranched PIB is equivalent to $J_{N}$ of linear PIB.

The temperature dependence of the linear viscoelastic properties was found to be the same for all branched and linear PIB. This equivalence of the temperature dependences is consistent with a model of Graessley (1982) for polymers having trans and gauche conformers of equal energy. Our results are at odds with an alternative viewpoint, based on the coupling model of relaxation [Santangelo et al. (1998)], which predicts that entangled branches will always confer greater temperature sensitivity, due to enhanced intermolecular cooperativity [Ngai and Roland (1997)]. However, the viscosity enhancement in these hyperbranched materials is not large (Fig. 5). Further study is warranted, particularly with branched PIB having greater viscosity enhancement over their linear counterpart.

Implicit in the consideration of the temperature dependence of rheological properties is the assumption that the local friction factor is independent of branch structure. This is certainly expected for moderate levels of branching, and perhaps even for very highly branched structures, due to the countervailing effect of an increased number of chain ends [Berry and Fox (1968)].

Our motivation for exploring alternative polymer architectures is improved processing behavior. This entails the nonlinear viscoelastic response, which is known to be sensitive to branching. This aspect of hyperbranched PIB's rheology remains to be investigated.

\section{ACKNOWLEDGMENTS}

The work at NRL was supported by the Office of Naval Research, and that at the University of Western Ontario by the Natural Sciences and Engineering Research Council of Canada. C.G.R. expresses his gratitude for an ASEE-NRL postdoctoral fellowship.

\section{References}

Adachi, K., T. Wada, T. Kawamoto, and T. Kotaka, "Dielectric spectroscopy on dilute blends of polyisoprene/ polybutadiene: Effects of matrix polybutadiene on the dynamics of probe polyisoprene," Macromolecules 28, 3588-3596 (1995). 
Antonietti, M., T. Pakula, and W. Bremser, "Rheology of small spherical polystyrene microgels: A direct proof for a new transport mechanism in bulk polymers besides reptation," Macromolecules 28, 4227-4233 (1995).

Atilgan, A. R., T. Haliloglu, I. Bahar, and B. Erman, "Correlated fluctuations in polymer networks," Comput. Theor. Polym. Sci. 8, 55-59 (1998).

Ball, R. C. and T. C. B. McLeish, "Dynamic dilution and the viscosity of star polymer melts," Macromolecules 22, 1911-1913 (1989).

Bauer, B. J. and L. J. Fetters, "Synthesis and dilute-solution behavior of model star-branched polymers," Rubber Chem. Technol. 51, 406-436 (1978).

Bero, C. A. and C. M. Roland, "Terminal relaxations in linear and 3-arm star polyisoprenes," Macromolecules 29, 1562-1568 (1996).

Berry, G. C. and T. G. Fox, “The viscosity of polymers and their concentrated solutions," Adv. Polym. Sci. 5, 261-357 (1968).

Bick, D. K. and T. C. B. McLeish, "Topological contributions to nonlinear elasticity in branched polymers," Phys. Rev. Lett. 76, 2587-2590 (1996).

Carella, J. M., J. T. Gotro, and W. W. Graessley, "Thermorheological effects of long-chain branching in entangled polymer melts," Macromolecules 19, 659-667 (1986).

Doi, M. and S. F. Edwards, The Theory of Polymer Dynamics (Clarendon, Oxford, 1986).

Erman, B. and J. E. Mark, Structures and Properties of Rubberlike Networks (Oxford University Press, New York, 1997).

Farrington, P. J., C. J. Hawker, J. M. J. Fréchet, and M. E. Mackay, "The melt viscosity of dendritic poly(benzyl ether) macromolecules," Macromolecules 31, 5043-5050 (1998).

Ferry, J. D., Viscoelastic Properties of Polymers (Wiley, New York, 1980).

Fetters, L. J., W. W. Graessley, and A. D. Kiss, "Viscoelastic properties of polyisobutylene melts," Macromolecules 24, 3136-3141 (1991).

Fetters, L. J., A. D. Kiss, D. S. Pearson, G. F. Quack, and F. J. Vitus, "Rheological behavior of star-shaped polymers," Macromolecules 26, 647-654 (1993).

Fréchet, J. M. J., "Functional polymers and dendrimers-Reactivity, molecular architecture, and interfacial energy," Science 263, 1710-1715 (1994).

Graessley, W. W., "Effect of long branches on the flow properties of polymers," Acc. Chem. Res. 10, 332-339 (1977).

Graessley, W. W., "Effect of long branches on the temperature-dependence of viscoelastic properties in polymer melts," Macromolecules 15, 1164-1167 (1982).

Graessley, W. W. and S. F. Edwards, "Entanglement interactions in polymers and the chain contour concentration," Polymer 22, 1329-1334 (1981).

Graessley, W. W., T. Masuda, J. E. L. Roovers, and N. Hadjichristidis, "Rheological properties of linear and branched polyisoprene," Macromolecules 9, 127-141 (1976).

Grest, G., L. J. Fetters, and J. S. Huang, "Star polymers: Experiment, theory, and simulation," Adv. Chem. Phys. XCIV, 67-163 (1996).

Hadjichristidis, N., "Synthesis of Miktoarm star (Mu-Star) polymers," J. Polym. Sci., Part A: Polym. Chem. 37, 857-871 (1999).

Hawker, C. J., "Dendritic and hyperbranched macromolecules-Precisely controlled macromolecular architectures," Adv. Polym. Sci. 147, 113-160 (1999).

Hawker, C. J., P. J. Farrington, M. E. Mackay, K. L. Wooley, and J. M. J. Fréchet, "Molecular ball bearings: The unusual melt viscosity behavior of dendritic macromolecules," J. Am. Chem. Soc. 117, 4409-4410 (1995).

Hutchings, L. R. and R. W. Richards, "Synthesis of deuterobutadiene-butadiene $\mathrm{AB}_{2}$ and $\mathrm{AB}_{3}$ Miktoarm star copolymers,' Polym. Bull. 41, 283-289 (1998).

Inkson, N. J., T. C. B. McLeish, O. G. Harlen, and D. J. Groves, "Predicting low density polyethylene melt rheology in elongational and shear flows with 'pom-pom' constitutive equations," J. Rheol. 43, 873-896 (1999).

Jacob, S. and J. P. Kennedy, "Synthesis, characterization and properties of octa-arm polyisobutylene-based star polymers," Adv. Polym. Sci. 146, 1-38 (1999).

Janzen, J. and R. H. Colby, 'Diagnosing long-chain branching in polyethylenes," J. Mol. Struct. 486, 569-584 (1999).

Kennedy, J. P., L. R. Ross, and O. Nuyken, “New telechelic polymers and sequential copolymers by polyfunctional initiator-transfer agents (Inifers). 14. Characterization of linear and three-arm star polyisobutylenes by degradative oxidation,'” Polym. Bull. 5, 5-10 (1981).

Kraus, G. and J. T. Gruver, "Rheological properties of multichain polybutadienes," J. Polym. Sci., Part A: Polym. Chem. A 3, 105-122 (1965).

Kwak, S.-Y. and H. Y. Lee, "Molecular relaxation and local motion of hyperbranched poly(ether ketone)s with reference to their linear counterpart. 1. Effect of degrees of branching," Macromolecules 33, 5536-5543 (2000). 
Long, V. C., G. C. Berry, and L. M. Hobbs, "Solution and bulk properties of branched polyvinyl acetates IV-Melt viscosity,', Polymer 5, 517-525 (1964).

MacArthur, A. and H. L. Stephens, "Reinforcement as a hindrance in the disentanglement of unknotted chains,' J. Appl. Polym. Sci. 28, 1561-1572 (1983).

McGrath, K. J., C. M. Roland, and M. Antonietti, "NMR study of the morphology of polystyrene linear/ microgel blends,', Macromolecules 33, 8354-8360 (2000).

McKenna, G. B., B. J. Hostetter, N. Hadjichristidis, L. J. Fetters, and D. J. Plazek, “A study of the linear viscoelastic properties of cyclic polystyrenes using creep and recovery measurements,' Macromolecules 23, 1834-1852 (1989).

McLeish, T. C. B. and R. G. Larson, "Molecular constitutive equations for a class of branched polymers: The pom-pom polymer,', J. Rheol. 42, 81-110 (1998).

McLeish, T. C. B., J. Allgaier, D. K. Bick, G. Bishko, P. Biswas, R. Blackwell, B. Blottiêre, N. Clarke, B. Gibbs, D. J. Groves, A. Hakiki, R. K. Heenan, J. M. Johnson, R. Kant, D. J. Read, and R. N. Young, "Dynamics of entangled H-polymers: Theory, rheology, and neutron scattering,' Macromolecules 32, 6734-6758 (1999).

Milner, S. T. and T. C. B. McLeish, "Parameter-free theory for stress relaxation in star polymer melts,' Macromolecules 30, 2159-2166 (1997).

Namba, S., Y. Tsukahara, K. Kaeriyama, K. Okamoto, and M. Takahashi, “'Bulk properties of multibranched polystyrenes from polystyrene macromonomers: Rheological behavior I,' Polymer 41, 5165-5171 (2000).

Ngai, K. L. and C. M. Roland, "Terminal relaxation and diffusion of entangled three-arm star polymers: Temperature and molecular weight dependences,', J. Polym. Sci., Polym. Phys. Ed. 35, 2503-2510 (1997).

Pakula, T., D. Vlassopoulos, G. Fytas, and J. Roovers, "'Structure and dynamics of melts of multiarm polymer stars," Macromolecules 31, 8931-8940 (1998).

Pearson, D. S., "Recent advances in the molecular aspects of polymer viscoelasticity,' Rubber Chem. Technol. 60, 439-496 (1987).

Pearson, D. S. and E. Helfand, "Viscoelastic properties of star-shaped polymers," Macromolecules 17, 888895 (1984).

Plazek, D. J. and I. Echeverría, “Don’t cry for me Charlie Brown, or with compliance comes comprehension,' J. Rheol. 44, 831-841 (2000).

Plazek, D. J., X. D. Zheng, and K. L. Ngai, "Viscoelastic properties of amorphous polymers. 1. Different temperature dependence of segmental relaxation and terminal dispersion,' Macromolecules 25, 4920-4924 (1992).

Puskas, J. E. and M. Grasmüller, 'Star-branched and hyperbranched polyisobutylenes,' Macromol. Symp. 132, 117-126 (1998).

Puskas, J. E., and C. Paulo, "Synthesis of hyperbranched polyisobutylenes by Inimer-type living polymerization. I. Investigation of the effect of reaction conditions,' Macromolecules 34, 734-739 (2001).

Puskas, J. E. and C. I. Wilds, “Multiarm-star polyisobutylenes by living carbocationic polymerization,' J. Polym. Sci., Polym. Chem. Ed. 36, 85-92 (1998).

Puskas, J. E., W. Pattern, P. M. Wetmore, and V. Krukonis, “Synthesis and characterization of novel multiarmstar polyisobutylene-polystyrene thermoplastic elastomers,'” Rubber Chem. Technol. 72, 559-568 (1999).

Roland, C. M. and C. A. Bero, "Normal mode relaxation in linear and branched polyisoprene,' Macromolecules 29, 7521-7526 (1996).

Roland, C. M., C. A. Bero, K. L. Ngai, and M. Antonietti, “Segmental relaxation in crosslinked rubber,' Mater. Res. Soc. Symp. Proc. 411, 367-374 (1996).

Roland, C. M., P. G. Santangelo, M. Antonietti, and M. Neese, "Mechanical behavior of polystyrene microgels,' Macromolecules 32, 2283-2287 (1999).

Roovers, J., "'Melt rheology of H-shaped polystyrenes,', Macromolecules 17, 1196-1200 (1984)

Roovers, J. and W. W. Graessley, "Melt rheology of some model comb polystyrenes,' Macromolecules 14, 766-773 (1981).

Roovers, J. and P. M. Toporowski, "Relaxation by constraint release in combs and star-combs," Macromolecules 20, 2300-2306 (1987)

Roovers, J., L. L. Zhou, P. M. Toporowski, M. Vanderzwan, H. Iatrou, and N. Hadjichristidis, "Regular star polymers with 64 and 128 arms-models for polymeric micelles,' Macromolecules 26, 4324-4331 (1993).

Santangelo, P. G. and C. M. Roland, "Effect of long-chain branching on the rheology of 1,4-polyisoprene,' J. Non-Cryst. Solids 235, 709-716 (1998).

Santangelo, P. G., K. L. Ngai, and C. M. Roland, "The coupling model approach to the terminal relaxation," Polymer 39, 681-687 (1998).

Santangelo, P. G., C. M. Roland, and J. E. Puskas, “Rheology of star-branched polyisobutylene,’ Macromolecules 32, 1972-1977 (1999).

Schroeder, M. J. and C. M. Roland, "Normal mode relaxation of polyisoprene in blends with vinyl polybutadienes," Macromolecules 32, 2000-2003 (1999).

Shull, K. R., K. H. Dai, E. J. Kramer, L. J. Fetters, M. Antonietti, and H. Sillescu, “Diffusion by constraint release in branched macromoleculer matrices,' Macromolecules 24, 505-509 (1991). 
Shull, K. R., E. J. Kramer, G. Hadziioannou, M. Antonietti, and H. Sillescu, "Diffusion of macromolecular stars in linear, microgel, and network matrices," Macromolecules 21, 2578-2580 (1988).

Storey, R. F., K. A. Shoemake, J. W. Mays, and S. Harville, "Synthesis and characterization of multiarm star-branched polyisobutylenes: Effect of arm molecular weight," J. Polym. Sci., Part A: Polym. Chem. 35, 3767-3778 (1997).

Suter, U. W., E. Saiz, and P. J. Flory, “Conformational characteristics of polyisobutylene," Macromolecules 16, 1317-1328 (1983).

Tead, S. F., E. J. Kramer, G. Hadziloannou, M. Antonietti, H. Sillescu, P. Lutz, and C. Strazielle, "Polymer topology and diffusion: A comparison of diffusion in linear and cyclic/macromolecules," Macromolecules 25, 3942-3947 (1992).

White, J. L., "Rheology of elastomers,” Rubber Chem. Technol. 42, 257-338 (1969).

Zhou, L.-L., N. Hadjichristidis, P. M. Toporowski, and J. Roovers, "Synthesis and properties of regular star polybutadienes with 32 arms," Rubber Chem. Technol. 65, 303-314 (1992).

Zimm, B. H. and W. Stockmayer, "The dimensions of chain molecules containing branches and rings," J. Chem. Phys. 17, 1301-1314 (1949). 